\title{
EM Detection of Viruses in Organ Transplant Patients
}

\author{
Sara E. Miller and David N. Howell
}

Department of Pathology, Duke University, Durham, NC 27710

Due to immunosuppression, transplant patients are more susceptible to infections, particularly viruses. The strengths of EM detection include the facts that it is rapid, non-invasive or minimally invasive, does not require probes, and can detect many different viruses. A limitation is that is it not as sensitive as PCR, but this can be an advantage in instances where endogenous viruses in healthy individuals may be detected by exquisitely sensitive tests. Additionally, concentration methods, such as ultracentrifugation, ultrafiltration, or immunoaggregation can increase the chances of finding them by EM in fluids, and localization of focal pathology in tissues can be enhanced by semithin sections, on-slide embedment of H\&E-stained sections, confocal microscopy, and laser capture.

In renal transplant patients, the types of viruses that may be seen by negative staining in urine include polyomavirus, adenovirus, small round viruses (SRV), such as those in the enterovirus family, and herpesviruses, such as cytomegalovirus. In our small bowel transplant patient population, 8 of 13 pediatric patients have had episodes of viral gastroenteritis diagnosed by EM. Viruses observed in this group are adenovirus, rotavirus, and small round structured viruses (SRSV), such as astrovirus, calicivirus, and Norwalk virus. Patients with bone marrow/stem cell transplants are profoundly immunodeficient. Viruses observed in fluids from this population are the same as in other transplant patients, e.g., adenovirus and enteroviruses from several sites, adenovirus and polyomavirus from urine, and adenovirus and SRSV, from stool. EM of thin sections of biopsies occasionally may reveal viruses.

Diagnostic pitfalls in the use of EM to detect viral pathogens can result from an absence of virus in the specimen submitted, because infections may exist in organs not transplanted but susceptible due to immunosuppression. Additionally, some viruses are not shed in effluents as they infect organs without contact with body cavities. Other types may remain cell-associated, i.e., stick to cell membranes, or incorporate viral DNA into the host genome and no longer produce recognizable virions. Incorporated "provirus" may also cause neoplasms, e.g., Epstein Barr virus (EBV) causes post transplant proliferative disease, a B-cell lymphoma, and Human herpes virus (HHV)-8 causes Kaposi sarcoma.

Proper specimen preparation is important in detecting viruses. Useful procedures to enhance visualization in fluids include concentrating the specimen, using hydrophilic support films, and removing cell debris by low speed centrifugation. Tissue samples can be prepared by routine embedding procedures or by rapid methods, including use of small tissue slivers and high heat resin curing and/or by microwave techniques.

Clues to virus identification in fluids include numerous small particles of similar size/shape, darker stain around the particle as opposed to a simple white spot, a fringe around the outside of amorphous-shaped particles, or filaments with a herringbone pattern $[1,2,3,4]$. Viruses may be naked icosahedral particles (Fig. 1a, b) or enveloped with a pliable membrane (Fig. 1c). Nucleocapsids inside enveloped viruses may be spherical (like Fig. 1a, b), helical (Fig. 1c), complex, or nondescript. Confusing things that look like viruses in fluids include small round organelles, such as exosomes, or droplets, such as lipids. Filamentous things, such as mucoprotein, may resemble filamentous nucleocapsids of the myxoviruses. 
Clues to virus identification in tissues include numerous small particles of similar size/shape, paracrystalline arrays (beware of glycogen), round particles in the nucleus (beware of nuclear pores), vesicles with regular-sized dark centers (beware of neurosecretory vesicles), and particles budding from cellular membranes $[1,2,3,4]$. Other structures that look like viruses in tissues are round organelles such as clathrin-coated or Golgi vesicles. Filamentous organelles in tissue, e.g., microtubules, intermediate filaments, and tubuloreticular inclusions, may be confused with helical nucleocapsids. With some exceptions, DNA viruses are usually constructed in the nucleus (Fig. 2a), and RNA viruses are made in the cytoplasm (Fig. 2b). Enveloped viruses usually bud from cell membranes (Fig. 2b inset).

In summary, all transplant patients are immunosuppressed and susceptible to virus infection. Diagnosis is imperative because the therapy for infection is diametrically opposed to that for organ rejection. Clues to virus identification include numerous sub-cellular particles of similar size/shape that differ from normal cellular organelles, but attention should be paid to possible look-alikes.

[1] SE Miller. J Electr Microsc Tech 4 (now J Micros Res Tech) (1986) p 265.

[2] FW Doane, N Anderson. Electron Microscopy in Diagnostic Virology, A Practical Guide and Atlas (Cambridge Univ Press, New York) (1987) 178 pp.

[3] Palmer EL, Martin ML. Electron Microscopy in Viral Diagnosis (CRC Press, Boca Raton) (1988) $194 \mathrm{pp}$.

[4] MA Hayat, SE Miller. Negative Staining (McGraw-Hill, New York) (1991) 253 pp.

[5] CS Goldsmith, SE Miller. Clin Microbiol Rev 22 (2009) p 552.
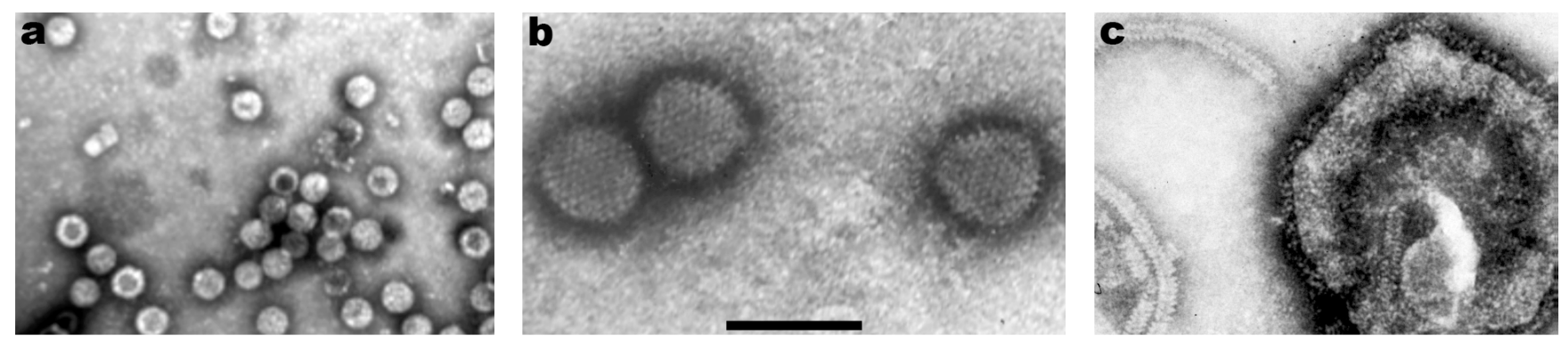

Fig.1. Negative stains. a. SRV (naked); b. adenovirus (naked); c. paramyxovirus (enveloped), spiked virion (right), helical nucleocapasids (left). Bar $=100 \mathrm{~nm}$ in a-c.
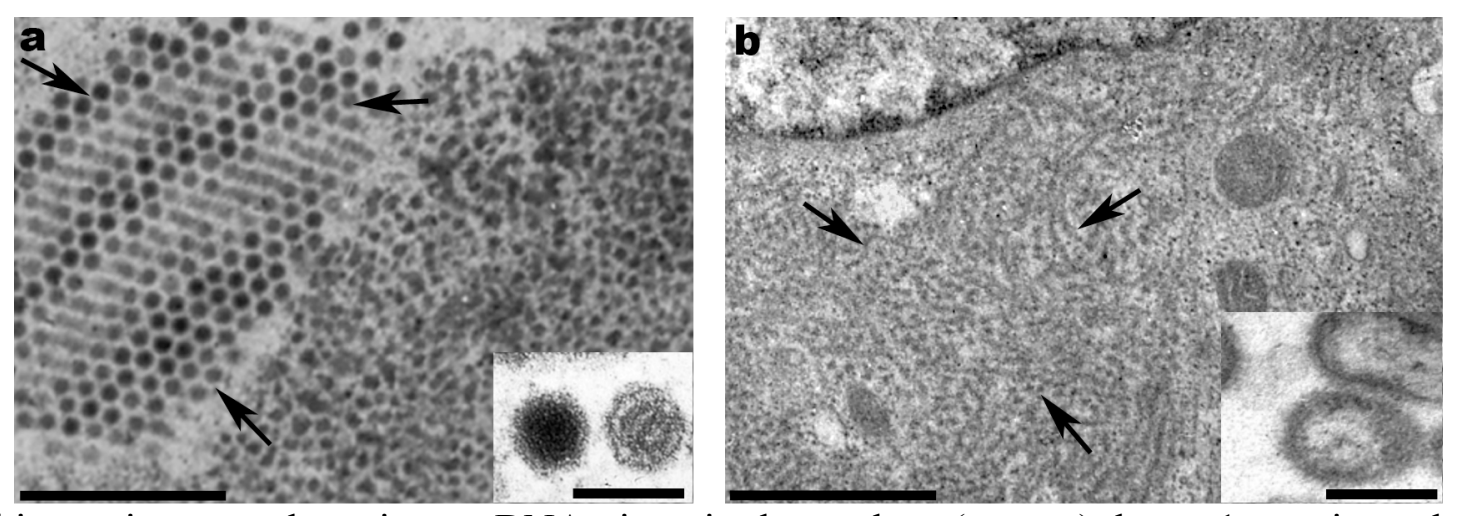

Fig. 2. Thin sections. a. adenovirus, a DNA virus, in the nucleus (arrows), bar $=1 \mu \mathrm{m}$; inset, bar $=100$ $\mathrm{nm}$; b. paramyxovirus nucleocapsids, an RNA virus, in the cytoplasm (arrows), bar $=1 \mu \mathrm{m}$; inset, complete enveloped virions (fuzz around the outside corresponds to the spikes in Fig. 1c), bar $=100 \mathrm{~nm}$. 